\title{
FRICTION COEFFICIENT OF A DISK IN A SHEET OF VISCOUS FLUID: NUMERICAL CALCULATION
}

\author{
J.R. HERINGA, F.W. WIEGEL \\ Department of Applied Physics, Twente University of Technology, P.O. Box 217, Enschede, \\ The Netherlands \\ and \\ F.P.H. van BECKUM \\ Department of Applied Mathematics, Twente University of Technology, P.O. Box 217, Enschede, \\ The Netherlands
}

Received 12 March 1981

\begin{abstract}
The translational friction coefficient of a porous cylindrical disk moving in a sheet of fluid which is embedded on both sides in a fluid of different viscosity, is calculated numerically for a wide range of the relevant parameters. These results are used to evaluate the region of validity of certain asymptotic formulae which have been derived previously in the limit of small ratios of the viscosities.
\end{abstract}

\section{Introduction}

During the last five years the following problem has arisen in the context of cell membrane hydrodynamics: calculate the drag force on a protein that performs a lateral Brownian motion in a cell membrane $\left.{ }^{1}\right)$. An asymptotic solution of this problem has been presented by $S_{a f f} \operatorname{man}^{2}$ ), who used the following model. The protein is represented by a cylindrical disk (radius $a$ and height $h$ ) and the cell membrane by a plane sheet (thickness $h$ ) of fluid with a viscosity $\eta$. The disk is constrained to move in this sheet. The sheet is embedded on both sides in another fluid with a viscosity $\eta^{\prime}$. Saffman has derived an approximate expression for the drag force, which holds asymptotically in the limit in which $\eta h \gg \eta^{\prime} a$. The first aim of the present paper is to estimate the accuracy of Saffman's asymptotic formula by comparing it with the results of a numerical calculation.

A very similar hydrodynamic problem arises if one considers the Brownian motion of a patch of cross-linked proteins in a cell membrane ${ }^{3}$ ). In this case the same geometry and the same model can be used with one exception: the disk, which now represents the patch of proteins, is porous and has a constant permeability $k_{0}$. The flow of fluids through permeable media forms the subject 
of a recent monograph ${ }^{4}$ ) to which the reader is referred for full details. A combination of these methods with Saffman's asymptotic method again leads to an asymptotic formula for the drag force ${ }^{5}$ ). In this case the condition $\eta h \gg \eta^{\prime} a$ for the asymptotic result is not always satisfied in realistic cases. Hence again the need for a numerical treatment arises.

The contents of this paper are organized as follows. In section 2 we briefly summarize the general theory as developed in refs. 4 and 5 , and we reduce the problem to a set of coupled differential equations. In these equations two dimensionless parameters play a role:

$$
\begin{aligned}
\sigma & \equiv \frac{a}{\sqrt{\bar{k}_{0}}}, \\
\theta & \equiv \frac{\eta h}{\eta^{\prime} a} .
\end{aligned}
$$

The numerical solution of these equations for a rather wide range of $(\sigma, \theta)$ combinations forms the subject of section 3; the results are given in table I. Various concluding remarks are collected in the last section. In this paper we shall generally follow the notation of refs. 4 and 5 .

\section{Theoretical considerations}

Consider a Cartesian system of coordinates $(x, y, z)$ with the $z$-axis along the axis of the cylinder. Shortly we also use cylindrical coordinates $(r, \phi, z)$. The fluid with viscosity $\eta$ is located at $-h<z<0$; the fluid with viscosity $\eta^{\prime}$ is located at $z<-h$ and at $z>0$.

First, consider the pressure $p$ and velocity $v=\left(v_{x}, v_{y}, v_{z}\right)$ of the fluid in the half-space $z>0$. These should be solved from the linearized, time-independent Navier-Stokes equation and from the incompressibility equation under the boundary condition that at large distances from the $z$-axis $v_{x} \rightarrow-v_{0}, v_{y} \rightarrow 0, v_{z} \rightarrow 0$. It has been shown in refs. 4 and 5 that this flow has the form

$$
\begin{aligned}
& v_{x}=-v_{0}-r \frac{\partial s}{\partial r} \cos ^{2} \phi+s+r \frac{\partial s}{\partial r}, \\
& v_{y}=-r \frac{\partial s}{\partial r} \cos \phi \sin \phi, \\
& v_{z}=0, \\
& p=\text { constant. }
\end{aligned}
$$


Here $s(r, z)$ is an unknown function with cylindrical symmetry which vanishes if $r \rightarrow \infty$ or $z \rightarrow \infty$, and which is a solution of the partial differential equation

$$
\left(\frac{\partial^{2}}{\partial r^{2}}+\frac{3}{r} \frac{\partial}{\partial r}+\frac{\partial^{2}}{\partial z^{2}}\right) s(r, z)=0
$$

Second, consider the fluid in the sheet $-h<z<0$. In this sheet the fluid (which represents a lipid membrane in the fluid phase) consists of rod-like molecules parallel to the $z$-axis. Therefore the flow will be two-dimensional. In the presence of a porous cylinder one has to use the (space-time) average pressure $P$ and the average velocity $V$ of this flow. The flow pattern should now be solved from the Debye-Brinkman-Bueche equation in combination with the incompressibility equation, subject to the same boundary condition at infinity as $v$. It has been shown in refs. 4 and 5 that the solution has the form

$$
\begin{aligned}
& V_{x}=-v_{0}-r \frac{\mathrm{d} S}{\mathrm{~d} r} \cos ^{2} \phi+S+r \frac{\mathrm{d} S}{\mathrm{~d} r}, \\
& V_{\mathrm{y}}=-r \frac{\mathrm{d} S}{\mathrm{~d} r} \cos \phi \sin \phi \\
& V_{z}=0, \\
& P=\eta \Pi(r) \cos \phi .
\end{aligned}
$$

Here $S(r)$ and $\Pi(r)$ are two functions of $r$ only, which have to be solved from the coupled equations

$$
\begin{aligned}
& -\Pi^{\prime}+S^{\prime \prime}+\frac{3}{r} S^{\prime}-\frac{S}{k}+\frac{v_{0}}{k}+\frac{2 \eta^{\prime}}{h \eta}\left(\frac{\partial s}{\partial z}\right)_{z=0}=0, \\
& -r \Pi^{\prime \prime}-\Pi^{\prime}+\frac{\Pi}{r}=\frac{a}{k_{0}}\left(v_{0}-S(a)\right) \delta(r-a), \\
& k(r)=k_{0}, \quad r<a, \\
& k(r)=\infty, \quad r>a .
\end{aligned}
$$

The prime denotes differentiation with respect to $r$. The function $S(r)$ is related to the function $s(r, z)$ by

$$
S(r)=s(r, 0) \text {. }
$$

Eqs. (13), (14) express the fact that the porous disk has a constant permeability $k_{0}$. 
Note that (12) can be solved to give

$$
\begin{aligned}
& \Pi(r)=L r, \quad r<a, \\
& I(r)=L a^{2} / r, \quad r>a,
\end{aligned}
$$

where $L$ is a constant which can be determined by integrating both sides of (12) from $r=0$ to $r=\infty$; this gives

$$
L=\frac{v_{0}-s(a, 0)}{2 k_{0}} \text {. }
$$

The $x$-component of the total force $F$ which the fluid exerts on the disk is given by

$$
F=\frac{+\eta}{k_{0}} \int v_{x}(r) \mathrm{d}^{3} r
$$

where the integral extends over the volume of the $\operatorname{disk}^{4}$ ). Substitution of (7)-(9) gives

$$
F=-\frac{\pi \eta h a^{2}}{k_{0}}\left(v_{0}-s(a, 0)\right)
$$

Note that this formula expresses the total force on a permeable disk in terms of the value of the function $s(r, z)$ at the circumference of the disk. This is similar to the Faxén theorem for the force exerted by a fluid on a porous sphere which has recently been derived by Felderhof and Jones ${ }^{6}$ ). Combination of (20) with (18) gives

$$
F=-2 \pi \eta h a^{2} L \text {. }
$$

Hence the friction coefficient $f$ is given by

$$
f=2 \pi \eta h a^{2} L / v_{0},
$$

and the diffusion coefficient follows from the Einstein relation

$$
D=\frac{k_{\mathrm{B}} T}{f},
$$

where $T$ is the absolute temperature and $k_{\mathrm{B}}$ Boltzmann's constant.

\section{Numerical analysis and results}

In order to calculate the drag on the disk one has to solve (6) subject to the boundary condition (11) at $z=0$. The other boundary conditions are: (i) $\partial s / \partial r=0$ at $r=0$; (ii) $s(\infty, z)=0$; (iii) $s(r, \infty)=0$. It is convenient to use the 
dimensionless variables $\rho \equiv r / a, \zeta \equiv z / a, \lambda \equiv L k_{0} / v_{0}$ and $\psi \equiv s / v_{0}$, in terms of which (11) reads

$$
\begin{aligned}
& \frac{\partial^{2} \psi}{\partial \rho^{2}}+\frac{3}{\rho} \frac{\partial \psi}{\partial \rho}+\frac{2}{\theta} \frac{\partial \psi}{\partial \zeta}+\sigma^{2}(1-\lambda-\psi)=0, \quad 0<\rho<1, \zeta=0, \\
& \frac{\partial^{2} \psi}{\partial \rho^{2}}+\frac{3}{\rho} \frac{\partial \psi}{\partial \rho}+\frac{2}{\theta} \frac{\partial \psi}{\partial \zeta}+\frac{\lambda \sigma^{2}}{\rho^{2}}=0, \quad 1<\rho, \zeta=0 .
\end{aligned}
$$

For numerical treatment it is desirable to transform the first quadrant of the $\rho, \zeta$ plane into a unit square. This can be accomplished in various ways; a convenient transformation is

$$
\begin{aligned}
& \tilde{\rho}=\frac{1}{2}\left(\frac{\rho}{1+\rho}+\frac{\rho}{\theta+\rho}\right), \\
& \tilde{\zeta}=\frac{1}{2}\left(\frac{\zeta}{1+\zeta}+\frac{\zeta}{\theta+\zeta}\right) .
\end{aligned}
$$

In these variables (7) can be written in the form

$$
\frac{\partial}{\partial \tilde{\rho}}\left(\rho^{3} \frac{\partial \tilde{\rho} / \partial \rho}{\partial \tilde{\zeta} / \partial \zeta} \frac{\partial \psi}{\partial \tilde{\rho}}\right)+\frac{\partial}{\partial \tilde{\zeta}}\left(\rho^{3} \frac{\partial \tilde{\zeta} / \partial \zeta}{\partial \tilde{\rho} / \partial \rho} \frac{\partial \psi}{\partial \tilde{\zeta}}\right)=0 .
$$

The transformation of the boundary conditions to these new variables is also straightforward.

The unit square is covered by an equally spaced rectangular grid and the "box-integration" method of Wachspress") is applied to replace (27) by a set of coupled linear equations. These are solved with the ICCG (3) algorithm of

TABLE I

\begin{tabular}{|c|c|c|c|c|c|c|c|}
\hline 9 & 0.1 & 0.5 & 1. & 2. & 5. & 10. & 100 \\
\hline 1 & 0.004993 & 0.1207 & 0.437 & 1.27 & 2.8 & 3.5 & 3.8 \\
\hline $5 / 3$ & 0.004990 & 0.1189 & 0.414 & 1.10 & 2.1 & 2.5 & 2.7 \\
\hline 3 & 0.004985 & 0.1164 & 0.386 & 0.92 & 1.6 & 1.8 & 1.9 \\
\hline 7 & 0.004978 & 0.1123 & 0.345 & 0.72 & 1.1 & 1.1 & 1.2 \\
\hline 15 & 0.004970 & 0.1083 & 0.310 & 0.58 & 0.78 & 0.84 & 0.86 \\
\hline 29 & 0.004962 & 0.1049 & 0.283 & 0.49 & 0.63 & 0.67 & 0.68 \\
\hline 61 & 0.004953 & 0.1011 & 0.257 & 0.42 & 0.52 & 0.54 & 0.55 \\
\hline 125 & 0.004944 & 0.0976 & 0.235 & 0.37 & 0.44 & 0.46 & 0.46 \\
\hline
\end{tabular}

Numerical results for the translational friction coefficient in units $2 \pi \eta h$, for various values of the dimensionless variables $\sigma=a / \sqrt{k_{0}}$ and $\theta=h \eta / a \eta^{\prime}$. The numerical accuracy is expected to be about one unit in the last decimal place 
Meyerink and Van der Vorst $t^{8}$ ) for $\lambda=0$ and $\lambda=1$. The correct value of $\lambda$ now follows from (18). Finally the friction coefficient can be calculated from (22). Calculations were performed on grids containing up to $32 \times 32$ lattice points, sometimes $64 \times 64$ for comparison. In table I we give the value of $f / 2 \pi \eta h$ thus calculated for various values of $\sigma$ and $\theta$.

\section{Concluding remarks}

The numerical values of the translational friction coefficient, tabulated in table 1, should be compared with the asymptotic formula

$$
\frac{f}{2 \pi \eta h} \approx 2\left\{-\gamma+\ln \theta+\frac{2}{\sigma^{2}}+\frac{I_{0}(\sigma)}{\sigma I_{1}(\sigma)}\right\}^{-1}, \quad \theta \gg 1,
$$

which has been derived by one of $\left.u^{4,5}\right)$. Here $\gamma \simeq 0.5772$ denotes Euler's constant and the $I_{\nu}$ are the modified Bessel functions. Approximate values calculated from the last formula are tabulated in table II. Comparison of the two tables shows: (1) that the asymptotic formula is accurate for $\theta \geqslant 15$ to within about $2 \%$; (2) that the asymptotic formula, where in error, overestimates the friction coefficient $f$, hence underestimates the diffusion coefficient $D$.

A calculation for a similar problem has been presented by Jones et al. ${ }^{9}$ ). They consider the diffusion of a polymer along the interface between two fluids of different viscosity. In that model the interface could represent a membrane, which is infinitely thin and does not have a hydrodynamics of its own.

TABLE II

Approximate results for $f / 2 \pi \eta h$, from the asymptotic formula (28) which holds for $\theta \gg 1$

\begin{tabular}{|c|c|c|c|c|c|c|}
\hline$\theta$ & 0.1 & 0.5 & 1. & 2. & 5. & 10. \\
\hline $5 / 3$ & 0.004998 & 0.1236 & 0.479 & 1.74 & 8.42 & 33.88 \\
\hline 3 & 0.004990 & 0.1193 & 0.420 & 1.15 & 2.42 & 3.09 \\
\hline 7 & 0.004980 & 0.1135 & 0.356 & 0.77 & 1.20 & 1.34 \\
\hline 15 & 0.004970 & 0.1088 & 0.314 & 0.60 & 0.82 & 0.89 \\
\hline 29 & 0.004962 & 0.1051 & 0.284 & 0.50 & 0.65 & 0.69 \\
\hline 61 & 0.004953 & 0.1011 & 0.257 & 0.42 & 0.52 & 0.55 \\
\hline 125 & 0.004944 & 0.0976 & 0.236 & 0.37 & 0.44 & 0.46 \\
\hline
\end{tabular}


The striking discrepancy between the experimental ${ }^{10}$ ) values for the lateral diffusion coefficients of proteins in membranes and the diffusion coefficients calculated from (28) can, therefore, not be the result of poor convergence of the asymptotic formula. We hope to discuss the biophysical consequences and physical limitations, like interface irregularity, of our calculation elsewhere ${ }^{11}$ ).

Finally we would like to remark that a similar numerical calculation could be performed for the rotational friction coefficient, for which an asymptotic expression has been derived too $^{12}$ ). However, as the rotational transport coefficients of proteins in cell membranes are less important than the translational ones such a calculation will probably be of little interest.

\section{References}

1) P.G. Saffman and M. Delbrück, Proc. Natl. Acad. Sci. USA 72 (1975) 3111.

2) P.G. Saffman, J. Fluid Mech. 73 (1976) 593.

3) F.W. Wiegel, J. Theor. Biol. 77 (1979) 189.

4) F.W. Wiegel, Lecture Notes In Physics 121 (Springer, New York, 1980).

5) F.W. Wiegel, J. Phys. A12 (1979) 2385.

6) B.U. Felderhof and R.B. Jones, Physica 93A (1978) 457.

7) E.L. Wachspress, Iterative Solution of Elliptic Systems and Applications to the Neutron Diffusion Equations of Reactor Physics (Prentice Hall, New York, 1966).

8) J.A. Meyerink and H.A. van der Vorst, Math. Comput. 31 (1977) 148.

9) R.B. Jones, B.U. Felderhof and J.M. Deutch, Macromolecules 8 (1975) 680.

10) W.W. Webb, in: Frontiers of Biological Energetics, Vol. 2 (Academic Press, New York, 1978).

11) J.R. Heringa and F.W. Wiegel, to be published.

12) F.W. Wiegel, Phys. Lett. 70A (1979) 112. 